\title{
Bibliometric Analysis of Top 100 Cited Articles on Prader-Willi Syndrome
}

\author{
Xiangying Li ${ }^{1,2}$, Hongbo Yang ${ }^{1}$, Linjie Wang ${ }^{1}$, Huijuan Zhu ${ }^{1}$, Fengying Gong ${ }^{1}$ and Hui Pan*1 \\ ${ }^{1}$ Department of Endocrinology, Key Laboratory of Endocrinology of National Health Commission, Peking Union Medical College Hospital, \\ China \\ ${ }^{2}$ Department of Endocrinology, China
}

*Corresponding author: Hui Pan, Department of Endocrinology, Key Laboratory of Endocrinology of National Health Commission,

Peking Union Medical College Hospital, Beijing, China

\begin{abstract}
ARTICLE INFO
Received: 蔧 March 09, 2019

Published: 唪 March 18, 2019

Citation: Xiangying Li, Hongbo Yang, Linjie Wang1, Huijuan Zhu, Fengying Gong, Hui Pan. Bibliometric Analysis of Top 100 Cited Articles on Prader-Willi Syndrome. Biomed J Sci \& Tech Res 16(1)-2019. BJSTR. MS.ID.002799.
\end{abstract}

Keywords: Prader-Willi Syndrome; Bibliometricmetric

\section{ABSTRACT}

There is an amount of articles published on Prader-Willi Syndrome (PWS). Bibliometric analysis is applied to provide insights into the most impactful studies in the field. In December 2018, we used the Thomson Reuters Web of Science to search the articles published, relating to Prader-Willi Syndrome. Articles were analyzed using the sequent characteristics: citation number, publication year, study design, country of origin, authorship and journal. Keyword analysis and citation networks were used to analyze research trends. $47 \%$ of the most cited articles were published in 1990s. The average number of Total Citations (TCs) was 183.09 (90-804) and the average number of Annual Citations (ACs) was 9.33(5.29-51.29). Among top 100 most-cited articles, 83 were original articles. Research mainly focused on genetics heredity (56\%).More articles were published in American Journal of Human Genetics , taking up $11 \%$ of the top 100 articles. Most of the articles were from USA (70\%). The top-ranked article (804 citations) was authored by Holm VA et al. in 1993. And Nicholls RD had 16 articles in the top 100 cited articles . Bibliometric analysis may offer deep insights into the research developing process and the analysis results can point out the future research focus.

\section{Introduction}

Prader-Willi Syndrome (PWS) is a rare genetic disease and its incidence was reported from $1 / 10,000$ to $1 / 25,000$ births $[1,2]$. There are three main mechanisms, which lead to absence of expression of paternally inherited genes in the 15q11.2-q13 region: paternal microdeletion, maternal uniparental disomy and imprinting defect [3]. Accredited genetic testing is the key to early diagnosis and treatment. Developmental delay, cognitive disability, and behavior problems usually become apparent in childhood. Many clinical manifestations of PWS are associated with hypothalamic dysfunction [4]. Recombinant Human Growth Hormone (rhGH) and/or gonadosteroid hormones replacement therapy would be advantageous to patients [2]. PWS has a significant impact on quality of life and represents a heavy financial strain on family and society. To decipher the characteristics of significant studies of PWS and point out the future research focus, bibliometric analysis was performed.

\section{Methods}

\section{Search Strategy}

We performed a title-specific search of the Web of Science (WOS) database (Thomson Reuters, New York, USA) to identify highly cited articles. Search expressions term was "Prader-Willi Syndrome" without restricting publication dates and language. As no patients were enrolled, ethical approval was not required.

\section{Data Extraction and Bibliometric Parameters}

"Prader-Willi Syndrome" were searched within the publication years ranged from 1972 to 2018 in titles, abstracts, author keywords. This search approach substantially augments title-word 
and author-keyword searching. Then the data were analyzed for the article title, authors, journal of publication, original country, year of publication and citation.

\section{Results}

\section{Article Analysis}

Our query produced 2458 articles published the field of PWS according to WOS. English was the most frequently used language (2388, 97.15\%). The United States (994, 40.44\%), England (184, $7.49 \%)$, Italy $(172,7.00 \%)$, Netherlands $(139,5.66 \%)$ and Japan $(133,5.41 \%)$ were the top five countries publishing the most number of articles on PWS. Most of the articles were published in American Journal of Human Genetics $(175,7.11 \%)$, American Journal of Medical Genetics (164, 6.67\%), American Journal of Medical Genetics Part A (125, 5.08\%) and Journal of Clinical Endocrinology Metabolism (72, 2.93\%). For further analysis, we focused on detailed aspects of the top 100 articles. Total citation numbers of top 10 ranged from 286 to 804 .

\section{Year of Publication}

According to the publication date range, we performed an analysis of publication trends by 10 -year intervals , while $47 \%$ were published in 1990s. PWS is a gene disorder, as studies thus far indicate that the complete phenotype is due to the loss of expression of several genes. 19 high cited articles of PWS in 1990s were genetics research conjunction with Angelman Syndrome (AS) which represent perhaps the best examples of genomic imprinting in humans. The specific genetic etiology in individuals with PWS is essential for the genetic counselling of affected families .

\section{Citations}

The top 100 most-cited articles received a total of 18836 citations .The average number of Total Citations (TCs) was 183.09 (90-804). The average number of articles Annual Citations (ACs) was 9.33(5.29-51.29). The trends of in TCs and ACs of the mostcited articles were not exactly matched. Six of the top 10 articles according to TCs were ranked in the top 10 articles list according to ACs. "Prader-Willi Syndrome "by Cassidy published in Genetics in Medicine received the highest AC (51.29) while its TC was 359. Syndrome The most commonly cited article (804 citations) was "Prader-Willi Syndrome- Consensus Diagnostic-Criteria" by Holm, Va (1993) in Paediatrics. This article was a consensus of diagnosticcriteria .It provided two scoring systems: one for children aged 0 to 36 months and another one for children aged three to adults. These criteria helped in identification of the syndrome in hypotonic infants and in adolescents and adults who were obese, mildly retarded, behaviourally disturbed. They also ensured uniform diagnosis for clinical and laboratory research in PWS.

\section{Article Type and Research Focus}

Among the top 100 most cited articles, 83 were original articles,11 were review articles. The rest are letters [4] and proceeding paper [2]. Main research categories include genetics heredity (56\%), biochemistry molecular biology (16\%), pediatrics (14\%), endocrinology metabolism (14\%), general internal medicine (8\%). Based on a more detailed classification method, research were mainly focused on genetic mechanism (54\%), body composition (11\%), cognitive ability and quality of life (6\%), treatment with hormone replacement therapy (5\%), diagnostic tests (4\%), epidemiology (3\%) and unclassified aspects (4\%). The rest of the high cited articles gave an overview of PWS (13\%) . Prader-Willi syndrome (PWS) and Angelman syndrome (AS) are neurogenetic disorders that are caused by the loss of function of imprinted genes in 15q11-q13. Twenty-nine of the top cited articles which simultaneously studied PWS and AS represented a paradigm for understanding the relationships between genome structure, epigenetics, evolution, and function. As many scientists hypothesized that ghrelin levels may play a role in the expression of obesity in this syndrome. Three of top 100 did ghrelin involved research. Furthermore, the relationship between ghrelin and GH secretion were of considerable current research interest. Nowadays the greatest challenge is to define clinical guidelines for better outcomes for PWS individuals.

\section{Journal of Publication}

The top 100 most cited articles were published in 41 journals. Among these journals, American Journal of Human Genetics published the greatest number (11\%), followed by American Journal of Medical Genetics (8\%), Human Molecular Genetics (8\%), Journal of Clinical Endocrinology Metabolism (8\%), Nature Genetics (8\%) . Obviously, most of these journals are specialized in the field of genetics.

\section{Countries for Publication}

A total of 26 countries were responsible for the top 100 mostcited articles. Most of the articles were from USA (70\%), followed by England (20\%), Germany (12\%), France (7\%) and Canada (7\%).

\section{Authors}

The top 100 articles were contributed by 409 authors. Nicholls RD had 16 articles in the most cited articles and an average citation of 388

\section{Conclusion}

It was clear that literature related to the absence of expression of imprinted gene (s) were mainstream. PWS not only has a significant impact on quality of life but also represents a heavy financial strain on family and society. What we can do is to help subjects with PWS form a good habit of regularly exercising, managing food environment and considering of group home placement in adulthood when necessary. This study provided a systematic overview of research work and the findings could be used for prioritizing future research efforts in PWS research. 


\section{References}

1. Briegel W (2018) Neuropsychiatric aspects of Prader-Willi syndrome-A review. Z Kinder Jugendpsychiatr Psychother 46: 238-246.

2. Burman P, Ritzen EM, Lindgren AC (2001) Endocrine dysfunction in Prader-Willi syndrome: A review with special reference to GH. ENDOCR REV 22: 787-799.

\section{ISSN: 2574-1241}

DOI: 10.26717/BJSTR.2019.16.002799

Hui Pan. Biomed J Sci \& Tech Res

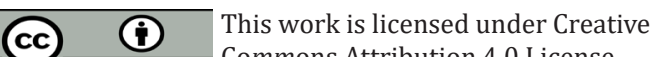
Commons Attribution 4.0 License

Submission Link: https://biomedres.us/submit-manuscript.php
3. Butler MG, Hartin SN, Hossain WA, Manzardo AM, Kimonis V, et al (2018) Molecular genetic classification in Prader-Willi syndrome: A multisite cohort study. J Med Genet 56(3): 149-153.

4. Emerick JE, Vogt KS (2013) Endocrine manifestations and management of Prader-Willi syndrome. Int J Pediatr Endocrinol, p. 1-14.

$\begin{array}{ll}\text { BIOMEDICAL } & \text { Assets of Publishing with us } \\ \text { RESEARCHES } & \text { - Global archiving of articles } \\ & \text { - Immediate, unrestricted online access } \\ & \text { - Rigorous Peer Review Process } \\ \end{array}$

